\title{
Credit Token based Rental Protocol for Dynamic Channel Allocation
}

\author{
D. Grandblaise ${ }^{*}$, K. Moessner ${ }^{\dagger}$, G. Vivier ${ }^{*}$ and R. Tafazolli ${ }^{\dagger}$ \\ "Motorola Labs, Parc Les Algorithmes, Commune de Saint Aubin, 91193 Gif sur Yvette, France, Email: \\ \{david.grandblaise,guillaume.vivier\}@motorola.com \\ †University of Surrey, GU2 7XH Guilford, United Kingdom, \\ Email: \{k.moessner, r.tafazolli\}@surrey.ac.uk
}

\begin{abstract}
This paper describes a distributed, cooperative and real time rental protocol for DCA operations in a multi system and multi cell context for OFDMA systems. A credit token based rental protocol using auctioning is proposed in support of dynamic spectrum sharing between cells. The proposed scheme can be tuned adaptively as a function of the context by specifying the credit tokens usage in the radio etiquette. The application of the rental protocol is illustrated with an ascending bid auctioning. The paper also describes two approaches for BS-BS communications in support of the rental protocol. Finally, it is described how the proposed mechanisms contribute to the current approaches followed in the IEEE $802.16 \mathrm{~h}$ and IEEE 802.22 standards efforts addressing cognitive radio.
\end{abstract}

Keywords: Dynamic Channel Allocation (DCA), rental protocol, auctioning, radio etiquette, cognitive radio, policy

\section{INTRODUCTION}

Space time varying spectrum usage [1] motivates for dynamic spectrum sharing between different radio access systems operated by different operators. In this manner, spectrum can be dynamically and temporally reused for a secondary usage by secondary systems when not used by the primary one. Secondary spectrum usage can be achieved with distributed dynamic channel allocation (DCA) in which spectrum is pooled [2] between cells and can be accessed by any of the systems in a geographical area where cells of the different systems overlap or are neighbours. Spectrum sharing with such a DCA in multi system and multi cell environment can be reached provided frequency reuse distances between the primary and secondary cells is appropriately managed to deal with co-channel interference. The force of distributed DCA is that it uses local information about the current available channels in each cell offering more suitable conditions to reuse frequency as often as possible. With this approach, the spectrum availability knowledge can be performed on peer to peer basis between neighbouring or overlapping cells.

In this multi cell and system environment enabling secondary spectrum usage with DCA, one main challenge is to schedule primary cell's radio resources (time + frequency) between several secondary cells competing for the access and usage of these resources while ensuring access fairness. One way to achieve this is to facilitate "real time automated negotiation of leased use rights" [3] for spectrum sharing between primary and secondary cells in a distributed and real time fashion. With respect to this, in this paper, we propose the negotiation is approached with a real time rental protocol using distributed and dynamic auctioning between each primary cell and secondary cells participating to DCA. In such a local multi cell environment market place, the primary cell acts as the resource offeror, and the secondary cells act as the resource renters. The primary cell (blue cell in the middle in Figure 1) opens for renting its resources for a temporally use for the secondary cells belonging to different operators (secondary cells are dashed, and each operator has two neighbouring cells with the primary cell in this example).

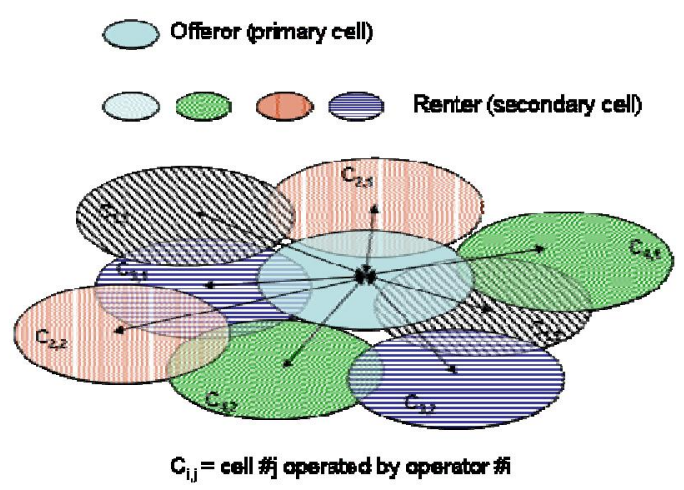

Figure 1 : Primary and secondary cells in DCA

The application of auctioning for resources sharing for cognitive radios has been initially proposed in the specific case of spectrum pooling [4]. This has been further elaborated in [5] and [6]. However, these proposed schemes consider auctioning between several users (cognitive radios enabled) competing to resources access and usage within a same cell. These papers do not address spectrum sharing between cells.

The present paper extends this by designing a distributed and cooperative rental protocol for DCA operations in a multi system and multi cell context for OFDMA systems. 
This paper is organised as follows. Section II introduces the credit token based rental protocol and radio etiquette for DCA. Section III describes how this rental protocol can be implemented for an ascending auctioning strategy. Section IV discusses two options enabling BS-BS communications in support of the rental protocol. Section $\mathrm{V}$ describes how the proposed mechanisms contribute to the current approaches followed in the IEEE 802.16h and IEEE 802.22 standards efforts. Finally, Section VI concludes the paper.

\section{CREDIT TOKEN BASED RENTAL PROTOCOL AND RADIO ETIQUETTE}

\section{A. Credit Token Based Rental Protocol Between Cells}

This section describes the main mechanisms of the renting protocol relying on credit token usage. It is assumed that the different secondary cells are owned by different operators (Figure 1). It should be noted that depending on the space time traffic variations, cell can periodically act as a primary or secondary cell.

The resources open for renting depends on the radio access technology under consideration. In this paper, we focus on OFDMA based systems. OFDMA provides the required flexibility to allocate dynamically in the temporal and frequency domain (continuously and fragmentally). With respect to this, let a BIN (one OFDM symbol * subchannel) be the smaller radio resource unit that can be offered for renting by the primary cell.

Let's assume that the primary cell can advertise to its neighbouring cells that it offers for renting a given amount of $\mathrm{X}$ BINs for a given period $\Delta$. This cell proposes a reserved price auction (RPA) for this renting. RPA is expressed as a number of credit tokens per BIN and per time unit (X is normalised to $\Delta$ ). Based on this RPA, each secondary cell can bid with a given amount of credit tokens per BN ( $\geq$ RPA) for $X^{\prime}$ BINs $\left(X^{\prime} \leq X\right)$ and for a period $\Delta^{\prime} \leq \Delta$. The auctioning period is defined as the period during which negotiation occurs. This period has a time duration $\delta$. It can be periodical.

We introduce and define the credit token as the quantum unit used for the negotiation transaction (selling, purchase, awarding) in any considered auctioning schemes between one primary cell and several secondary cells. Each cell is initially assigned with a credit token budget. Credit token usage and budget limitation enable both to solve contention issues on BINs usage between competing secondary cells. Since a credit token charging is as a function of the required amount of BINs for a given period $\Delta^{\prime} \leq \Delta$ by the secondary cell, it provides access fairness for secondary spectrum reuse between different secondary cells.

Additionally, credit tokens usage provides the means to give incentive to each primary cell to share totally or partially their BINs if they are unused or underused. With respect to this, a number of credit tokens can be awarded to any primary cell as a function of the rented time and the amount of rented BINs per time unit. The primary cell will be able to use these credit tokens at the time it will act as a secondary cell.

Since resources sharing between the primary cell and secondary cell are agreed for a given period, the proposed approach guarantees a minimum access time to the secondary cell, so it is favourable to support QoS.

\section{B. Credit Token Based Rental Protocol and Radio Etiquette}

In this paper, we propose (Figure 2) that the radio etiquette rules the rental protocol between cells. This radio etiquette specifies the policies applied to credit tokens usage during the negotiation transactions.

This approach provides the flexibility to adapt, to tune the auctioning method to be used during the transactions as a function of the context. In particular, the choice of the auctioning method [7] can be tuned as a function of the following parameters for example: the number of bidders (i.e. the number of secondary cells participating to auctioning), the number of BINs offered for renting (i.e. as a function of the space time traffic fluctuations), the renting time duration $\Delta$, the auctioning period $\delta$, etc.

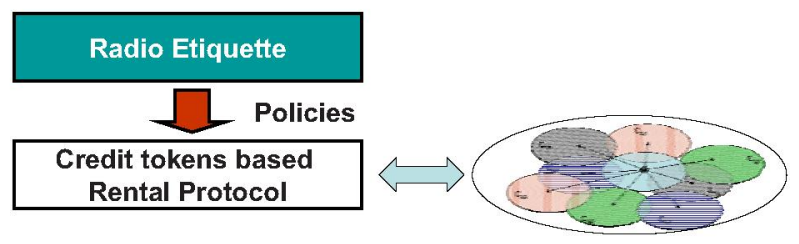

Figure 2 : Radio etiquette ruling the credit token based rental protocol via policies

This approach provides the means for an adaptive policy based dynamic resource sharing management. In particular, given the dynamic and real time aspects of the negotiation, software agents can be used at each BS (primary and secondary) to perform the negotiation during the auctioning period. Agents would supervise and apply the local rules specified by the radio etiquette. This approach paves the way towards a collaborative, dynamic, distributed and cognitive radio resource management between cells.

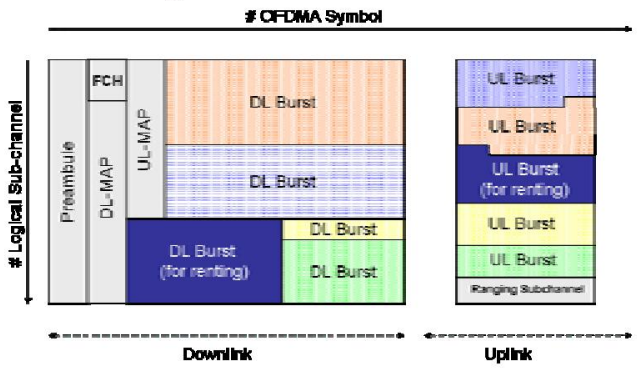

Figure 3: Example of OFDMA MAC frame structure for the radio resources (BNs) rental protocol 


\section{CREDIT TOKEN BASED RENTAL PROTOCOL EXAMPLE}

This section illustrates the application of the previous rental protocol for ascending bid auctioning in the case of an OFDMA frame structure (Figure 3).

\section{A. Rental Protocol Cycle}

The overall negotiation and transaction process between one primary BS (offeror) and several secondary BS (renter) can be depicted by the credit token based scheduling cycle (Figure 4 and Figure 5). For the sake of simplicity, these figures are only depicted for one secondary cell although several secondary cells are actually considered. The primary BS advertises about its auction. Provided this auction method, the interested secondary BSs can bid during a predefined period $(\delta)$ of negotiation specified by the primary cell. During this period, a dynamic and iterative process is launched to handle the ascending bid auctioning.

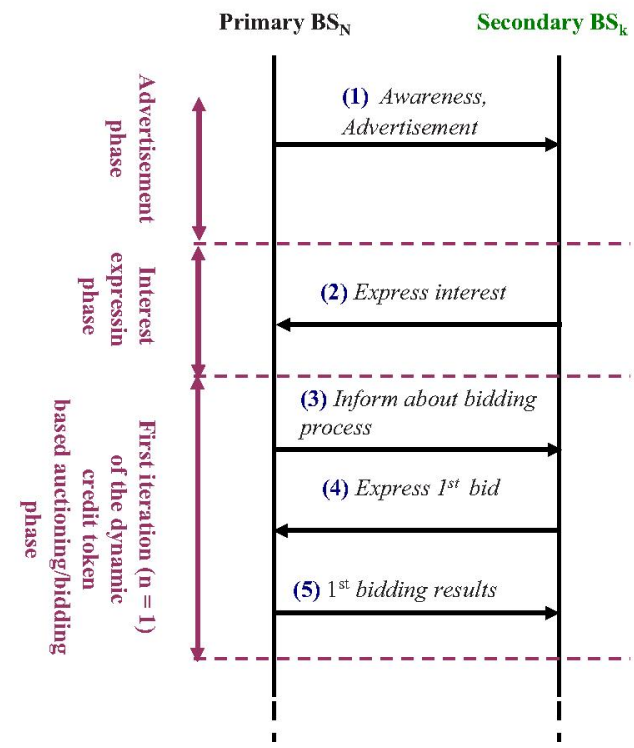

Figure 4: Credit token based scheduling cycle - (sequences (1) to (5))

\section{B. Description of the Rental Protocol Sequences}

This section describes a possible algorithm related to the different sequences of Figure 4 and Figure 5 (case of ascending bid auctioning).

In sequence $(1), \mathrm{BS}_{\mathrm{N}}$ advertises that $\mathrm{X}$ BNs are open for renting for a period $\Delta$ (from starting time $T_{\text {Start }}$ to ending time $\mathrm{T}_{\text {End }}$ ). $\mathrm{BS}_{\mathrm{N}}$ proposes a reserve price auction RPA for this renting. RPA is expressed as a number of credit tokens per BIN.

In sequence (2), each $\mathrm{BS}_{\mathrm{k}}$ informs $\mathrm{BS}_{\mathrm{N}}$ about its willingness (or not) to participate to the bidding. If the $\mathrm{BS}_{\mathrm{k}}$ is interested, it communicates its $i d_{k}$ to $\mathrm{BS}_{\mathrm{N}}$.
In sequence (3), $\mathrm{BS}_{\mathrm{N}}$ provides information about the auctioning period $\delta$ (and other policies) to the $\mathrm{BS}_{\mathrm{k}} \mathrm{s}$ that have expressed the interest to participate to the bidding. $\delta=\mathrm{T}_{\text {End }}$ Bidding $-T_{\text {Start Bidding. }}$

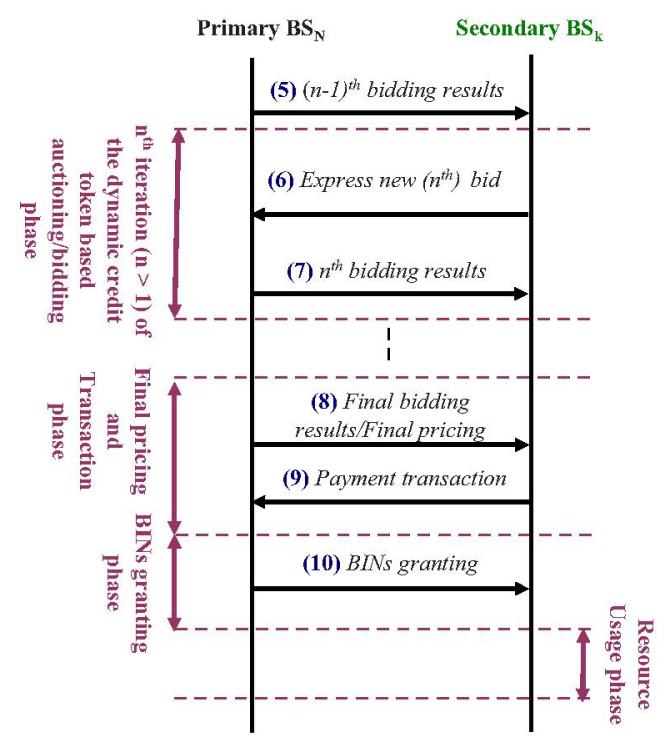

Figure 5: Credit token based scheduling cycle - (sequences (5) to (10))

In sequence (4), each $\mathrm{BS}_{\mathrm{k}}$ provides the information $\mathrm{BID}^{(1)}{ }_{\mathrm{k}}$ $=\left\{\mathrm{BS} \mathrm{CT}^{(1)}{ }_{\mathrm{k}}, \mathrm{X}_{\mathrm{k}}, \mathrm{T}_{\mathrm{Start} \mathrm{k}}, \mathrm{T}_{\text {End } \mathrm{k}}\right\}$ to $\mathrm{BS}_{\mathrm{N}} . \mathrm{BS} \mathrm{CT}^{(1)}{ }_{\mathrm{k}}$ is the amount of bidded credit tokens per BIN proposed by $\mathrm{BS}_{\mathrm{k}}$ for the first $(n=1)$ iteration. $X_{k}$ is the amount of BINs $\left(X_{k} \leq X\right)$ for which bid BS_CT ${ }^{(1)}{ }_{k}$ applies for. $\left[T_{\text {Start } k}, T_{\text {End } k}\right]$ is the time interval for which bid BS_CT ${ }_{k}^{(1)}$ applies for. [ $\mathrm{T}_{\text {Start } k \text {, }}$ $\left.\mathrm{T}_{\text {End } \mathrm{k}}\right] \subset\left[\mathrm{T}_{\text {Start }}, \mathrm{T}_{\text {End }}\right]$.

In sequence (5), $\mathrm{BS}_{\mathrm{N}}$ performs the following actions described hereafter. Given the set of intervals $\left\{\left[T_{\text {Start } k}, T_{\text {End }}\right.\right.$ $\mathrm{k}]\}$ received from different bidders $\left\{\mathrm{id}_{\mathrm{k}}^{(1)}\right\}, \mathrm{BS}_{\mathrm{N}}$ partitions $\left\{\left[\mathrm{T}_{\text {Start }}, \mathrm{T}_{\text {End }}\right]\right\}$ into contiguous time segments $\left\{\mathrm{TS}_{\mathrm{m}}\right\}$. Each $\mathrm{TS}_{\mathrm{m}}$ corresponds to a time window in which a subset of intervals of $\left\{\left[\mathrm{T}_{\text {Start } \mathrm{k}}, \mathrm{T}_{\mathrm{Endk}}\right]\right\}$ overlap. The different bidders $\left\{\mathrm{id}^{(1)}{ }_{\mathrm{k}}\right\}$ assigned to a given $\mathrm{TS}_{\mathrm{m}}$ are identified by $\left\{\mathrm{id}_{\mathrm{km}}^{(1)}\right\}$. $\left\{\mathrm{id}_{\mathrm{k}, \mathrm{m}}^{(1)}\right\}$ compete on $\mathrm{TS}_{\mathrm{m}}$. Each involved bidder $\mathrm{id}_{\mathrm{k}, \mathrm{m}}^{(1)}$ competes with its respective $\mathrm{BID}^{(1)}{ }_{\mathrm{k}}$. Then, for each $\mathrm{TS}_{\mathrm{m}}$, the $\mathrm{BS}_{\mathrm{N}}$ calculates the payoff $\mathrm{P}^{(1)}{ }_{\mathrm{k}}=\mathrm{BS} \mathrm{CT}^{(1)}{ }_{\mathrm{k}} * \mathrm{X}_{\mathrm{k}}$ for each bidder $\mathrm{k}$, and searches the subset $\left(\left\{\mathrm{id}_{\mathrm{k}, \mathrm{m}}^{(\overline{1})}\right\}_{\text {selected }}\right)$ of $\left\{\mathrm{id}_{\mathrm{k}, \mathrm{m}}^{(1)}\right\}$ such as $\operatorname{sum}\left(\mathrm{X}_{\mathrm{k}}\right)$ and $\operatorname{sum}\left(\mathrm{P}^{(1)}{ }_{\mathrm{k}}\right)$ are maximal. For each $\mathrm{TS}_{\mathrm{m}}$, $\mathrm{BS}_{\mathrm{N}}$ informs all $\left\{\mathrm{id}^{(1)}{ }_{\mathrm{k}, \mathrm{m}}\right\}$ about $\mathrm{P}^{\mathrm{min},(1)} \mathrm{m}$ and $\mathrm{P}^{\max ,(1)} \mathrm{m}$ where $\mathrm{P}^{\min ,(1)}{ }_{\mathrm{m}}$ is the minimal payoff from $\left\{\mathrm{id}^{(1)}{ }_{\mathrm{k}, \mathrm{m}}\right\}_{\text {selected }}$ and $\mathrm{P}^{\max \text {, }}$ (1) $\mathrm{m}$ is the maximal payoff from $\left\{\mathrm{id}^{(1)}{ }_{\mathrm{k}, \mathrm{m}}\right\}_{\text {selected }}$ during the $1^{\text {st }}$ iteration. With this approach, each $\mathrm{BS}_{\mathrm{k}}$ is directly informed whether it has been selected or not, and has some information on how far it is from $\mathrm{P}^{\mathrm{min},(1)} \mathrm{m}$ while still having some information on $\mathrm{P}^{\max ,(1)} \mathrm{m}$. This approach enables to keep the privacy of competing $\left\{\mathrm{id}_{\mathrm{k}, \mathrm{m}}^{(1)}\right\}$ on $\mathrm{TS}_{\mathrm{m}}$. 
In sequence (6), if $\mathrm{P}^{(1)}{ }_{\mathrm{k}}<\mathrm{P}^{\mathrm{min},(1)}{ }_{\mathrm{m}}$, this means that $\mathrm{BS}_{\mathrm{k}}$ has not been selected for being granted the resources he has bidded for during the first iteration $n=1$. More generally speaking, for $n>1$, if $\mathrm{P}^{(\mathrm{n}-1)}{ }_{\mathrm{k}}<\mathrm{P}^{\mathrm{min},(\mathrm{n}-1)} \mathrm{m}$, this means that $\mathrm{BS}_{\mathrm{k}}$ has not been selected for being granted the resources he has bided for during the $(\mathrm{n}-1)^{\text {th }}$ iteration. If $\mathrm{P}_{\mathrm{k}}^{(\mathrm{n}-1)}<\mathrm{P}^{\mathrm{min},(\mathrm{n}-1)} \mathrm{m}$ and if $\mathrm{BS}_{\mathrm{k}}$ is still interested to be allocated with the additional resources he initially requested for, it can propose a new BS CT ${ }_{k}^{(n)}$ for the $n^{\text {th }}$ iteration. Then, $\mathrm{BS}_{\mathrm{k}}$ computes the new $\mathrm{P}^{(\mathrm{n})}{ }_{\mathrm{k}}^{-}=\mathrm{BS} \mathrm{CT}^{(\mathrm{n})}{ }_{\mathrm{k}} * \mathrm{X}_{\mathrm{k}}$ where $\mathrm{X}_{\mathrm{k}}$ is fixed $\sqrt{\mathrm{n}}$ on $\mathrm{TS}_{\mathrm{m}}$. If $\mathrm{P}^{(\mathrm{n})}{ }_{\mathrm{k}}>$ $\mathrm{P}^{(\mathrm{n}-1)}{ }_{\mathrm{k}}$ and $\mathrm{P}^{(\mathrm{n})}{ }_{\mathrm{K}}>\mathrm{P}^{\mathrm{min},(\mathrm{n}-1)}{ }_{\mathrm{m}}, \mathrm{BS}_{\mathrm{k}}$ expresses its interest to keep on participating in the bidding with the new bid. In that case, it informs $\mathrm{BS}_{\mathrm{N}}$ with its new (update) value of $\mathrm{BS} \mathrm{CT}^{(\mathrm{n})}{ }_{\mathrm{k}}$. In case $\mathrm{P}^{(\mathrm{n})}{ }_{\mathrm{k}}=\mathrm{P}^{(\mathrm{n}-1)}{ }_{\mathrm{k}}$ or $\mathrm{P}^{(\mathrm{n})}{ }_{\mathrm{k}}<\mathrm{P}^{\mathrm{min},(\mathrm{n}-1)}{ }_{\mathrm{m}}, \mathrm{BS}_{\mathrm{k}}$ leaves the bidding phase and will not be granted with the additional resources he asked for.

In sequence (7), $\mathrm{BS}_{\mathrm{N}}$ updates $\left\{\mathrm{id}_{\mathrm{k}, \mathrm{m}}^{(\mathrm{n}-1)}\right\}$ into $\left\{\mathrm{id}_{\mathrm{k}, \mathrm{m}}^{(\mathrm{n})}\right\}$. Based on the new received biddings $\left\{\mathrm{BS} \mathrm{CT}^{(\mathrm{n})}{ }_{\mathrm{k}}\right\}$ for each $\mathrm{TS}_{\mathrm{m}}, \mathrm{BS}_{\mathrm{N}}$ calculates the new payoff $\mathrm{P}_{\mathrm{k}}^{(\mathrm{n})}=\mathrm{BS} \mathrm{CT}_{\mathrm{k}}^{(\mathrm{n})} * \mathrm{X}_{\mathrm{k}}$ for each bidder $\mathrm{k}$ who still participates to the bidding. Then, for each $\mathrm{TS}_{\mathrm{m}}, \mathrm{BS}_{\mathrm{N}}$ searches the subset $\left(\left\{\mathrm{id}_{\mathrm{k}, \mathrm{m}}^{(\mathrm{n})}\right\}_{\text {selected }}\right.$ ) of $\left\{\mathrm{id}^{(\mathrm{n})}{ }_{\mathrm{k}, \mathrm{m}}\right\}$ such as $\operatorname{sum}\left(\mathrm{X}_{\mathrm{k}}\right)$ and $\operatorname{sum}\left(\mathrm{P}^{(\mathrm{n})}{ }_{\mathrm{k}}\right)$ are maximal. Next, $\mathrm{BS}_{\mathrm{N}}$ performs the same actions as in sequence (5): for each $\mathrm{TS}_{\mathrm{m}}, \mathrm{BS}_{\mathrm{N}}$ informs all $\left\{\mathrm{id}^{(\mathrm{n})}{ }_{\mathrm{k}, \mathrm{m}}\right\}$ about $\mathrm{P}^{\mathrm{min},(\mathrm{n})}{ }_{\mathrm{m}}$ and $\mathrm{P}^{\mathrm{max},(\mathrm{n})}{ }_{\mathrm{m}}$ where $\mathrm{P}^{\mathrm{min},(\mathrm{n})}{ }_{\mathrm{m}}$ is the minimal payoff from $\left\{\mathrm{id}_{\mathrm{k}, \mathrm{m}}^{(\mathrm{n})}\right\}$ selected and $\mathrm{P}^{\max ,(\mathrm{n})}{ }_{\mathrm{m}}$ is the maximal payoff from $\left\{\mathrm{id}^{(\mathrm{n})}{ }_{\mathrm{k}, \mathrm{m}}\right\}_{\text {selected }}$ during the $n^{\text {th }}$ iteration. As long as $\delta>0$ (i.e. the auctioning period has not yet elapsed), $\mathrm{n}$ is increased and the mechanisms of the " $n$th iteration of the credit tokens based auctioning/bidding phase" keep on being applied.

In sequence (8), when $\delta=0$, bidding phase is over. None $\mathrm{BS}_{\mathrm{k}}$ can propose a new bid. $\left\{\mathrm{id}^{(\mathrm{n} \text { final })}{ }_{\mathrm{k}, \mathrm{m}}\right\}_{\text {selected }}$ is derived. At this point, $\mathrm{BS}_{\mathrm{N}}$ derives the clearing price auction $\mathrm{BS} \mathrm{CPA}_{\mathrm{k}}$ (expressed as a number of credit tokens per BIN) for each $\mathrm{TS}_{\mathrm{m}}$ and each $\mathrm{k}$ from $\left\{\mathrm{id}^{(\mathrm{n}}{ }^{\text {final) }}{ }_{\mathrm{k}, \mathrm{m}}\right\}$. For each $\mathrm{k}$ and $\mathrm{m}$, BS $\mathrm{CPA}_{\mathrm{k}}$ can correspond to the BS $\mathrm{CT}^{(\mathrm{final})}{ }_{\mathrm{k}}$, or can follow another price auction method.

In sequence (9), eack $\mathrm{BS}_{\mathrm{k}}$ is requested to pay $\operatorname{Pr}_{\mathrm{k}}=$ BS $\mathrm{CPA}_{\mathrm{k}} * \mathrm{X}_{\mathrm{k}}$ to be allowed to use the resources it won on its corresponding $\mathrm{TS}_{\mathrm{m}}$. Provided that $\operatorname{Pr}_{\mathrm{k}}$ does not exceed the credit tokens budget of $\mathrm{BS}_{\mathrm{k}}$, the credit tokens transaction between $\mathrm{BS}_{\mathrm{N}}$ and each $\mathrm{BS}_{\mathrm{k}}$ is performed.

In sequence $(10), \mathrm{BS}_{\mathrm{N}}$ grants the resource $\left(\mathrm{X}_{\mathrm{k}} \mathrm{BINs}\right)$ to each $\mathrm{BS}_{\mathrm{k}}$ who has successfully performed the credit tokens transaction operation in sequence $(9)$.

\section{INTER CELL COMMUNICATIONS}

This section discusses two options enabling BS-BS communications in support of the rental protocol.

\section{A. Possible Approaches}

The implementation of the rental protocol cycle can be enabled with the introduction of the appropriate signalling either over the backhaul (i.e. wired based), or over the air communications between BSs. In both cases, the usage of local databases (located at each BS) and regional databases (set of local databases) can be used to store the updated information about resources usage by each BS or regional area.

\section{B. Over the Backhaul}

In that case (Figure 6), the communications for the different phases (advertisement, bidding, etc) of the cycle between the primary and secondary BSs are carried out by the backhaul with IP based communications with the usage of a transaction server. The server acts as the gateway between primary and secondary BSs. This approach is appropriate for resource sharing negotiation which is not too sensitive to the delay (due to the latency introduced by the network).

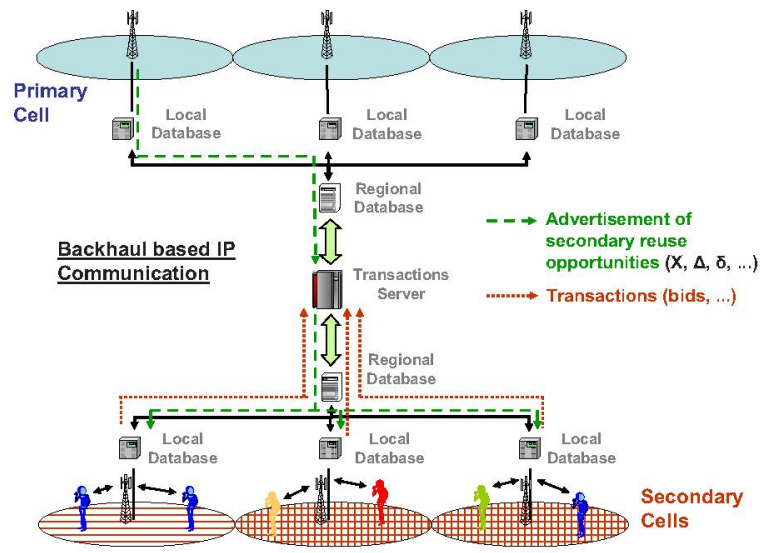

Figure 6: Signalling over the backhaul

\section{Over the Air}

In that case (Figure 7), the communications for the different phases (advertisement, bidding, etc) of the cycle between the primary and secondary BSs are carried out with over the air signalling in a distributed fashion. This means each BS can broadcast, multicast and communicate in peer to peer with its surrounding BSs depending on its state (primary or secondary) and the phase under consideration. By directly considering in built self healing functions and negotiation at the MAC layer, this approach is closer of a cognitive radio based solution (can integrate jointly sensing, allocation, negotiation functions). Additionally, it is less delay sensitive for real time transactions.

This over the air signalling can be physical or logical based. In case of physical based signalling, the communications is a RF link between the BSs. In case of logical based signalling, the over the air communications between BSs is established by the usage of a bridge (e.g. a terminal) that provides the RF links between BSs. [8] discusses how the credit token based rental protocol can be implemented with over the air physical signalling at the MAC layer. 


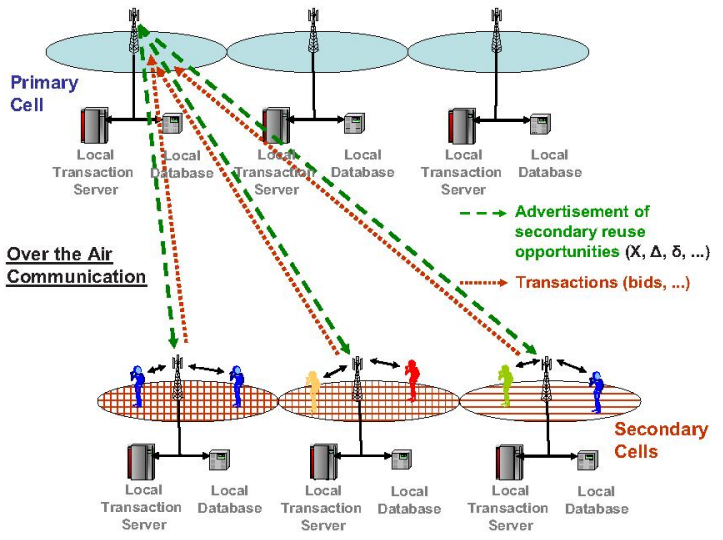

Figure 7: Over the air signalling between BS

\section{RENTAL PROTOCOL IN IEEE COGNITIVE RADIO STANDARDS}

The implementation of preliminary cognitive radio features in the design of a wireless air interface standard has started. Indeed, in the end of 2004, IEEE 802 has launched respectively the Project IEEE 802.16h [9] and IEEE 802.22 [10]-[11]. Purpose of 802.16 h Project is to specify improved MAC mechanisms in order: (i) to enable the co-existence among licensed exempt systems based on IEEE 802.16 standard, and (ii) to facilitate the co-existence of such systems with primary users. This amendment is applicable for un-coordinated frequency operation in all bands in which 802.16-2004 is applicable, including bands allowing shared services. Purpose of 802.22 is to allow the use of licensed exempt devices in the spectrum that is currently allocated in the television service. 802.22 targets to define both the PHY and MAC contrary to $802.16 \mathrm{~h}$ which can only modify the MAC of existing 802.16 specifications. Requirements for both $802.16 \mathrm{~h}$ and 802.22 are to reuse spectrum for primary systems but without causing any interference to incumbents. For both systems, two co-existence cases arise: co-existence with primary users, and self co-existence (i.e. co-existence among 802.16 systems themselves, or co-existence among 802.22 systems themselves). In support of the self coexistence, some proposals are being discussed in both projects to support dynamic spectrum sharing between 802.16 systems (respectively 802.22 systems). In particular, the introduction of rental and negotiation protocols for spectrum radio resources between BSs are under discussion

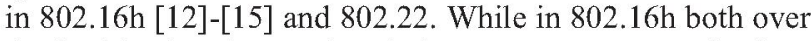
the backhaul and over the air inter system communications are under discussion, 802.22 focuses currently only on over the air approach.

\section{CONCLUSION}

This paper has described a distributed and real time rental protocol for DCA operations in a multi system and multi cell context for OFDMA systems. The proposed rental protocol is based on credit token transaction ensuring a collaborative secondary spectrum usage between primary and secondary cells. Different auctioning methods using the credit tokens can be designed as a function of the spectrum sharing context via the radio etiquette specifying policies. An illustration of the application of this rental protocol has been provided, and its relevance to IEEE $802.16 \mathrm{~h}$ and IEEE 802.22 standards has been pointed out. The proposed mechanisms pave the way towards a collaborative, distributed and cognitive radio resource management between cells. The protocol can be applied to licensed or unlicensed systems.

\section{ACKNOWLEDGEMENTS}

This work has been performed in the framework of the EU funded project $\left(\mathrm{E}^{2} \mathrm{R}\right.$ II). The authors would like to acknowledge the contributions of their colleagues from $E^{2} R$ II consortium.

\section{REFERENCES}

[1] New York City Spectrum Occupancy Measurements September 2004, December 15, 2004, Shared Spectrum Company.

[2] D. Grandblaise et al, "Extended DCA Paradigm for Distributed Licensed Open Spectrum Coordination", PIMRC 2005, 11-14 Sept, Berlin, Germany.

[3] ET Docket No. 03-108, Notice of Proposed Rule Making And Order, FCC, December 2003.

[4] Mitola, J., III; "Cognitive radio for flexible mobile multimedia communications", Mobile Multimedia Communications, 1999. (MoMuC 99), 15-17 Nov. 1999, pp.3-10.

[5] F.K. Jondral, C. Kloeck, H. Jaekel, "Auction Sequence as a New Resource Allocation Mechanism," IEEE VTC Fall 05, 2005.

[6] C. Kloeck, H. Jaekel, F.K. Jondral, "Dynamic and local combined pricing, allocation and billing system with cognitive radios, IEEE DySPAN 2005 Symposium, 8-11 Nov. 2005.

[7] V. Krishna; "Auction Theory", Academic Press 2002.

[8] D. Grandblaise, K. Moessner, G. Vivier, R. Tafazolli, "Credit token based scheduling for inter BS Spectrum Sharing", $4^{\text {th }}$ Karlsruhe Workshop on Software Radios (WSR'06), Karlsruhe, Germany, 22-23 March 2006.

[9] IEEE 802.16 License Exempt (LE) task Group (802/16h), http:/www.iece802.org/16/le/

[10] IEEE 802.22 Working Group on Wireless Regional Area Networks, http://www.ieee802.org/22/

[11] C. Cordeiros, K. Challapali, D. Birru and S. Shankar N, "IEEE 802.22: the First Worldwide Wireless standard based on Cognitive Radios", DySPAN 2005 Conference, 8-11 November, Baltimore, MD, USA.

[12] Contribution IEEE C802.16h-05/020r1, "Proposal for Credit Tokens Based Co-existence Resolution and Negotiation Protocol," D. Grandblaise, 11 July 2005.

[13] IEEE C802.16h - 05/036rl - "Proposal for enhanced credit tokens based co-existence resolution and negotiation protocol", D. Grandblaise, 08 November 2005.

[14] IEEE C802.16h - 05/037r1 - "Proposal for MAC frame mechanisms supporting enhanced credit tokens based co-existence resolution and negotiation protocol", D. Grandblaise, 08 November 2005.

[15] Working Draft Document for 802.16h (2006-02-06), IEEE 802.16h$06 / 005$. 